\title{
Cashflow como objeto virtual de aprendizaje de la Administración Financiera. Caso: Instituto Luis A. Martínez de Ambato
}

Cashflow as a virtual learning object of the Financial Administration. Case: Instituto Luis A. Martínez de Ambato

\begin{abstract}
Alexandra de los Ángeles Milla Estrada. ${ }^{1}$ \& Irma del Rocío Vázquez Andino. ${ }^{2}$
Recibido:01-10-2020 / Revisado: 25-10-2020 /Aceptado: 12-11-2020/ Publicado: 05-12-2020
\end{abstract}

Resumen.

DOI: $\underline{\text { https://doi.org/10.33262/concienciadigital.v3i4.1.1466 }}$

Introducción. La educación financiera representa un eje fundamental en la vida diaria de las personas; la administración efectiva de los recursos económicos conlleva a considerarse la creación de una sociedad más productiva y desarrollada; es por tal razón que los docentes de esta área están llamados a intervenir y aplicar nuevos paradigmas de enseñanza - aprendizaje de tal manera contrarrestar las ineficaces metodologías tradicionales. Objetivo. Demostrar que la utilización de Objetos Virtuales de Aprendizaje (OVAs) con escenarios semejantes a la realidad inciden positivamente en los educandos y facilitan las actividades de los docentes en el Instituto Tecnológico Luis A. Martínez. Metodología. Un enfoque cuantitativo, con la comprobación de hipótesis; la información se recolecto en dos instancias la primera con la aplicación del test de diagnóstico financiero, el test de inteligencias múltiples a una muestra de 106 estudiantes del establecimiento, concluyéndose con una entrevista semiestructurada realizada a cada docente de la especialidad; posteriormente se ejecuta un programático de entrenamiento del OVA (CASHFLOW) y se finaliza con test post- diagnósticos. Resultados. Entre los más significativos fue encontrar una diferencia del $40 \%$ entre el diagnóstico inicial y el post - diagnostico; con la aplicación del test financiero se evidenció que en principio tanto en conocimientos, actitudes y comportamiento el $60 \%$

\footnotetext{
${ }^{1}$ Instituto Superior Tecnológico Luis A. Martínez, Ambato, Ecuador. alexandramilla78@gmail.com https://orcid.org/0000-0001-7008-3217

${ }^{2}$ Universidad Europea del Atlántico Santander, España. doguiiva9@hotmail.com https://orcid.org/0000-0003-2626-2669
} 
presentaron resultados desfavorables en el área; en el test de inteligencias múltiples lo significativo de los sujetos analizados fue que demostraron tres tipos de inteligencias predominantes: intrapersonal, interpersonal y rítmica. Luego de la aplicación del programático, los resultados son significativamente favorables y todos los tipos de inteligencias múltiples analizadas presentan valores medianos. Conclusión. Se concluyó que la aplicación del objeto virtual de aprendizaje (CASFLOW) contribuye a mejorar las puntuaciones en el rendimiento académico y personal del estudiante; además estimula al docente a replantearse los métodos de enseñanza y evaluación que aplica en la actualidad.

Palabras clave: Paradigmas, OVAs, Administración Financiera, Aprendizaje.

\section{Abstract.}

Introduction. Financial education represents a fundamental axis in people's daily lives; the effective administration of economic resources leads to the creation of a more productive and developed society; It is for this reason that teachers in this area are called to intervene and apply new teaching-learning paradigms in such a way as to counteract the ineffective traditional methodologies. Objective. Demonstrate that the use of Virtual Learning Objects (OVAs) with scenarios similar to reality have a positive effect on students and facilitate the activities of teachers at the Luis A. Martínez Technological Institute. Methodology. A quantitative approach, with hypothesis testing; The information was collected in two instances, the first with the application of the financial diagnostic test, the multiple intelligences test to a sample of 106 students from the establishment, concluding with a semi-structured interview with each teacher of the specialty; subsequently, a programmatic OVA training program (CASHFLOW) is executed and ends with post-diagnostic tests. Results. Among the most significant was finding a difference of $40 \%$ between the initial diagnosis and the post-diagnosis; With the application of the financial test it was evidenced that in principle, both in knowledge, attitudes and behavior, $60 \%$ presented unfavorable results in the area; In the multiple intelligences test, the significance of the analyzed subjects was that they demonstrated three predominant types of intelligences: intrapersonal, interpersonal and rhythmic. After applying the programmatic, the results are significantly favorable and all the types of multiple intelligences analyzed present median values. Conclusion. It was concluded that the application of the virtual learning object (CASFLOW) contributes to improve the scores in the academic and personal performance of the student; It also encourages the teacher to rethink the teaching and assessment methods that they apply today.

Keywords: Paradigms, OVAs, Financial Management, Learning. 


\section{Introducción}

Actualmente la educación financiera representa un eje fundamental en la vida diaria de las personas; la administración efectiva de los recursos económicos que se adquiere desde muy joven, eleva el bienestar propio y de todas las personas que los rodean, lo que conlleva a considerarse la creación efectiva de una sociedad más productiva y desarrollada.

Para la gran mayoría de docentes de niveles medios, técnicos y universitarios en Latinoamérica, el instruir a los estudiantes con meras teorías de finanzas, contabilidad, matemáticas, entre otras materias de la educación financiera; es suficiente para que el estudiante pueda desenvolverse en la vida diaria y profesional; sin embargo, casi en su totalidad, fracasan cuando inician un emprendimiento, negocio o inversión. Estos acontecimientos emergen porque los estudiantes no obtuvieron prácticas en la vida real, no fueron preparados para ello y no se les motivo en alcanzar sus metas; afuera, cuando les toca defenderse por sí solos y quieren aplicar lo aprendido, les resulta un viacrucis mantener su pequeño negocio a flote; la mayoría desertan, caen en banca rota y por falta de trabajo o ingresos; gran número de estos jóvenes se unen a grupos delincuenciales, sumergiendo a su nación en el subdesarrollo.

Ecuador integra el grupo de países que aún se encuentran en crecimiento, pese a poseer grandes reservas naturales y capital humano dispuesto a producir, no ha encontrado el camino correcto para acercarse más al desarrollo. No hace falta hacer grandes investigaciones para concluir sobre la administración de un país, basta con observar cómo es el nivel de vida de sus habitantes para darse cuenta que, valores tan importantes como: la disciplina, respeto, responsabilidad, honestidad, empatía hacia los demás, actitudes positivas, entre otros valores que brinda una educación de calidad, sean considerados escasos o se goce de ellos.

El presente artículo demuestra la incidencia que provoca la utilización de metodologías de aprendizajes prácticos y de motivación personal, en un instituto de educación superior del Ecuador que ofrece carreras en administración financiera. En el estudio se analizó, se experimentó, evaluó y se concluyó sobre el uso de un objeto virtual de aprendizaje (OVA) que simuló un escenario real en el aprendizaje de la administración financiera, este objeto incluyó contenido de motivación para promover el emprendimiento. La hipótesis planteada pretendió demostrar que la utilización de OVAs con escenarios semejantes a la realidad, inciden positivamente en los educandos.

Las metodologías de enseñanza, se basan en teorías de psicología educativa, la mayoría de profesionales docentes han tratado o conocen teorías de psicólogos y pedagogos como Vygotsky, Gardner, Piaget Iván Pávlov, F.B. Skinner, Albert Bandura y Bloom quienes han realizado contribuciones significativas a la taxonomía de objetivos de la educación (MacDonald, 2019). 
Las siguientes son las teorías más comunes en el aprendizaje:

Conductismo. - Es la teoría que canaliza al alumno esencialmente como una "clean slate" o pizarra limpia para comenzar y moldeada por las emociones (MacDonald, 2019).

Cognitivismo. - La teoría cognitiva social generalmente se habla en el nivel de la primera infancia porque tiene que ver con el pensamiento crítico, siendo el concepto más importante la idea del juego. Se asume durante el proceso de aprendizaje que el pensamiento sigue una secuencia bastante común como: despertar el interés intelectual; exploración preliminar del problema; formulación de ideas, explicaciones o hipótesis; selección de ideas apropiadas; y verificación de su idoneidad (Peel, 2017).

Constructivismo. - Es un enfoque de la enseñanza y el aprendizaje basado en la premisa de que la cognición (aprendizaje) es el resultado de la "construcción mental". En otras palabras, los estudiantes aprenden ajustando nueva información junto con lo que ya saben. Los constructivistas creen que el aprendizaje se ve afectado por el contexto en el que se desarrolla una idea (BADA, 2015).

Teoría de las inteligencias múltiples. - Establece que las personas no necesitan ser inteligentes en todas las disciplinas para ser consideradas inteligentes, sino que las personas se destacan en varias disciplinas, lo que las hace excepcionales. Las inteligencias múltiples consideradas en la teoría son: musicales, espaciales, lingüísticos, matemáticos, kinestésicos, interpersonales, intrapersonales y naturalistas, cada persona tiene una inteligencia predominante y seguida de otra. (MacDonald, 2019).

Diseño universal para el aprendizaje (UDL). - Esta teoría se centra en cómo los maestros necesitan diseñar su plan de estudios para sus alumnos. se basa en la neurociencia y en cómo el cerebro procesa la información, realiza tareas y se entusiasma con la educación. Esta teoría incorpora a la tecnología en el aula y las formas de educar a los estudiantes en la era digital (MacDonald, 2019).

A partir de estas teorías los docentes pueden desarrollar otros estilos, métodos o técnicas de aprendizaje en función de la edad, niveles de educación, entorno educativo y estilos de aprendizaje de cada estudiante.

Con la aparición de la tecnología, se crean un sinnúmero de herramientas digitales que contribuyen y facilitan los aprendizajes; un gran número de estas herramientas contienen metodologías de enseñanza con entornos virtuales reales, es el caso de CASHFLOW una herramienta digital diseñada para el aprendizaje de la administración financiera y considerada para este estudio como un OVA. 
OVAs, - Hay varios filósofos que describen a los objetos de aprendizaje como elementos de instrucciones, donde el docente puede construir, armar, desarmar y adaptarlo a los diferentes contextos basados en la informática (Wiley, 2000).

No hay definición especifica de los OVAs el contexto nace por un paradigma constructivista y la necesidad de sistematizar el conocimiento, entorno a esto, depende de la motivación y capacidad del docente para que pueda crear y emplear elementos a fin de validar los aprendizajes (Latorre, 2006). Hay propiedades básicas que debe cumplir los objetos de aprendizaje:

Tabla 1. Propiedades básicas los objetos de aprendizaje en el aula

\begin{tabular}{|c|c|c|c|}
\hline \multicolumn{4}{|c|}{ Objeto de Aprendizaje } \\
\hline $\begin{array}{l}\text { Objetivo de } \\
\text { aprendizaje }\end{array}$ & $\begin{array}{l}\text { Contenido } \\
\text { Formativo }\end{array}$ & $\begin{array}{c}\text { Actividades de } \\
\text { aprendizaje }\end{array}$ & Autoevaluación \\
\hline
\end{tabular}

Fuente: (Maldonado, Bermeo, \& Vélez, 2017)

Estas propiedades no son un todo en las herramientas digitales, cada docente puede establecer un objetivo de clase y crear o adecuar la herramienta para cumplir su fin.

Antes de identificar la necesidad de un nuevo paradigma de enseñanza aprendizaje en el aula, el docente deberá aplicar test de conocimientos previos y habilidades; estas herramientas no son un todo, ni únicas; dependerá en gran medida de la habilidad del docente. Para este estudio específico se aplicó el test de diagnóstico y test de inteligencias múltiples.

Test de diagnóstico. - A medida que el estudiante va aprendido a través de la educación formal o informal, este tiende a construir sus propios puntos de vista, pero para el docente hay preocupación por las diferentes ideas o concepciones erróneos que algunos estudiantes pueden adoptar para la construcción de conceptos científicos; lo que puede impedir el aprendizaje o la identificación de componentes productivos; es por ello que hay la necesidad de las pruebas de diagnóstico, las mismas que son herramientas de evaluación que traen a la luz la diferencia entre lo que queremos que el alumno sepa o aprenda y lo que realmente sabe o aprende (Kaltakci, Eryılmaz, \& McDermot, 2015)

Test de inteligencias múltiples. - Se aplicó con la finalidad de conocer como el alumno aprende, y saber sobre las preferencias individuales, este test ayudó a comprender las formas de aprendizaje y adquisición de conocimiento (Sreenidhi \& Tay, 2017). El test aplicado determinó 8 capacidades metales de los estudiantes. 1. Inteligencia Lingüística. 2. Inteligencia Lógico - Matemática, 3. Inteligencia Visual - Espacial, 4. Inteligencia Kinestésica o Corporal-cinética, 5. Inteligencia Musical, 6. Inteligencia Interpersonal, 7. Inteligencia Intrapersonal, 8. Inteligencia Naturalista (Gardner, 2011). 
Una vez identificada la necesidad, se estableció estrategias para un aprendizaje significativo; en este caso se experimentó con un OVA, y antes de ponerlo en ejecución se preparó a la audiencia con técnicas pedagógicas de motivación, a fin de que se centren en el contenido y puedan interactuar con él; luego de observar, practicar, retroalimentarse y realizar las actividades de manera satisfactoria, se esperó que el estudiante adapte lo aprendido en su vida cotidiana y de resultados fructuosos.

Técnicas de motivación. - Antes de iniciar con la exposición de contenidos formativos en el aula, se preparó a los estudiantes a fin de captar el interés, motivarlos a participar y lograr la interactividad; en caso de investigación en otros grupos de interés, la motivación dependerá en gran medida de la habilidad del docente para lograr los objetivos de enseñanza.

Hay varios estudios realizados en torno a la motivación de los estudiantes de niveles superiores y los factores tomados en cuenta en esta investigación son:

\begin{tabular}{ll}
\hline FACTORES DE MOTIVACIÓN & DETALLE \\
\hline $\begin{array}{l}\text { Enlace entre los conocimientos previos y } \\
\text { los nuevos }\end{array}$ & $\begin{array}{l}\text { La relación de la nueva información con algún aspecto ya } \\
\text { existente en la estructura cognitiva del alumno. } \\
\text { El significado del material para el alumno } \\
\text { El material debe provocar ilusión, reto cognitivo y deseo de } \\
\text { conocer. }\end{array}$ \\
$\begin{array}{l}\text { La organización de la experiencia de } \\
\text { aprendizaje. }\end{array}$ & $\begin{array}{l}\text { Planificar el contenido en función de las inteligencias } \\
\text { múltiples de los estudiantes. } \\
\text { promover al estudiante para que sea protagonista y se } \\
\text { apropie del conocimiento, en donde su posición no se } \\
\text { reduzca a escuchar y repetir la materia. }\end{array}$ \\
Características del objetivo de estudio & Contenidos actualizados, claros, precisos y concisos. \\
\hline
\end{tabular}

Fuente: (Abarca, 1995)

Elaborado por: las autoras

Una vez preparada la audiencia, se inicia con la ejecución del programático; para este experimento se consideró como objeto virtual de aprendizaje el software CASHFLOW (flujo de caja), un juego de mesa virtual que propone la criticidad en el ámbito financiero, despierta la imaginación en la negociación e inversión y fomenta la planeación financiera personal.

CASHFLOW Tecnologies Inc. - Creado en 1996 por el empresario Robert Kiyosaki, quien, a partir de sus experiencias como inversionista, instruye y motiva a elevar el bienestar financiero de las personas (Kiyosaki, 2012). Características. - juego que gamifica la educación financiera diseñado para cualquier persona; forma en la compra y venta de bienes raíces, inversiones a corto y largo plazo, como obtener ganancias o flujo de caja, simula como manejar las oportunidades de inversión reales antes de desembolsar dinero, como manejar activos que fluyen en efectivo o ingresos pasivos; mediante el juego concientiza al estudiante sobre cómo es su comportamiento financiero en la vida real (Kiyosaki R. , 2019). 


\section{Metodología.}

El estudio tiene un enfoque cuantitativo; para la comprobación de la hipótesis se recolecto información en dos instancias; la primera con la aplicación del test de diagnóstico financiero y test de inteligencias múltiples a una muestra de 106 estudiantes del Instituto Tecnológico Luis A Martínez. El propósito fue medir el nivel y domino de conocimientos en administración financiera, analizar las fortalezas o el área de talento de los estudiantes, conocer que tan preparados están para su desarrollo como profesionales fuera de la institución; además, esta información contribuyo a construir una narrativa analítica de la lógica que guía la educación financiera en el establecimiento. La muestra elegida está caracterizada de la siguiente forma:

\section{Gráfico No. 1 Característica de la muestra según rango de edad}

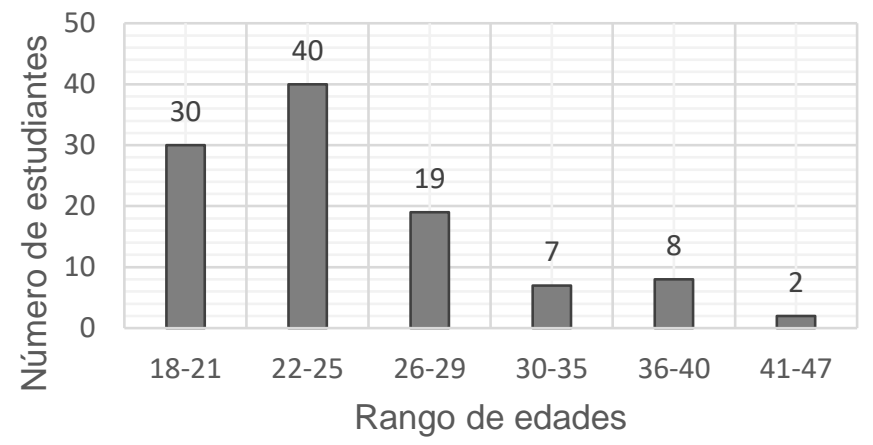

Fuente: trabajo de campo

Elaborado por: las autoras

Como se describe en el grafico existe una mayoría de estudiantes que están en un rango de edad entre los 22 y 25 años; hay que destacar que, dentro del grupo total, se observa la presencia de 17 estudiantes mayores de 30 años y 2 de ellos están entre los 41 y 47 años de edad.

\section{Gráfico No. 2 Característica de la muestra según sexo}

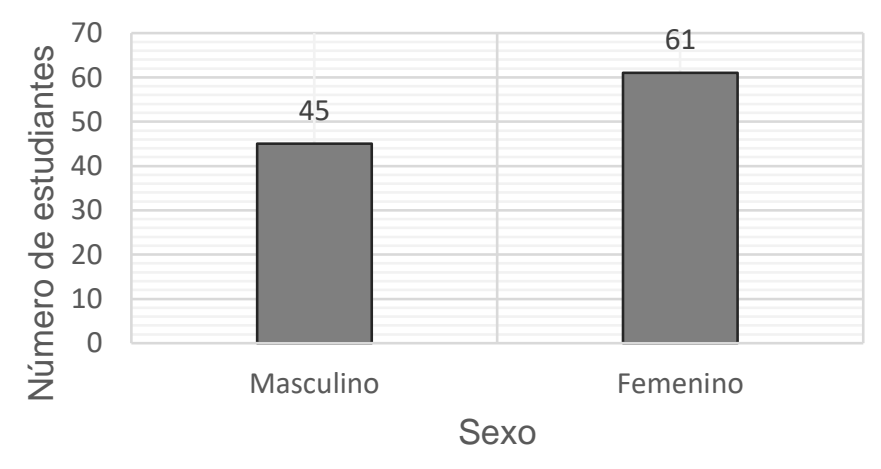

Fuente: trabajo de campo 
Elaborado por: las autoras

Como describe el grafico, la muestra está compuesta por 45 hombre y su mayoría está representada por 65 mujeres.

\section{Gráfico No. 3 Característica de la muestra según nivel de carrera}

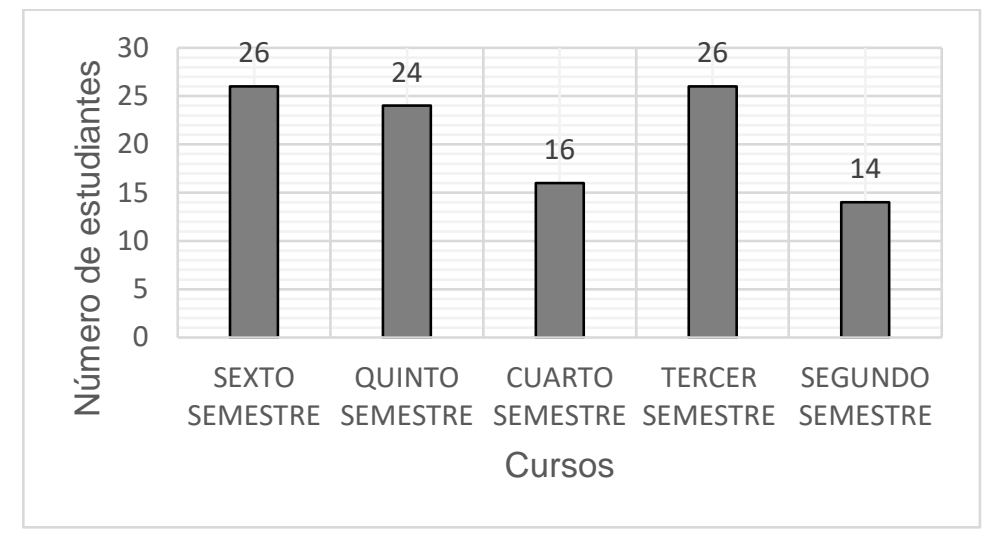

Fuente: trabajo de campo

Elaborado por: las autoras

La muestra está conformada por estudiantes de 5 niveles o cursos, como se observa se ha considerado estudiantes de sexto, quinto, cuarto, tercer y segundo semestre.

En este primer apartado se concluye con una entrevista semiestructurada realizada a cada docente de la especialidad; en referencia al proceso de enseñanza que sigue y ejecuta en el aula, metodologías y estrategias que utiliza; y el tipo de herramientas tecnológicas que están inmersas en estos procesos; todas estas consideradas como núcleo de la entrevista semiestructurada.

Una vez obtenido resultados de esta primera etapa, se llevó a cabo el entrenamiento de los estudiantes durante el periodo de 2 meses. Este tiempo se aprovechó para la concientización sobre la educación financiera personal e instrucción del OVA (CASHFLOW).

Después de esta etapa de entrenamiento y con la finalidad de comprobar la hipótesis, se aplicó un test post - diagnóstico de conocimientos técnicos financieros adquiridos en la etapa de entrenamiento y el test de inteligencias múltiples, que tenía como fin determinar el grado de motivación del estudiante en referencia a la educación financiera.

\section{Resultados.}

\section{Fase diagnóstica}

En el test de diagnóstico financiero se evalúa 3 componentes, el primero, los conocimientos técnicos financieros que dan como resultados el número de aciertos y errores; el segundo la actitud financiera, sus resultados demuestran que tan favorable o desfavorable esta la 
predisposición del estudiante en torno a sus finanzas y por último el comportamiento financiero, los resultados evidenciaran si la manera de comportarse frente a situaciones económicas es positiva o negativa.

\section{Gráfico No. 4 Componentes de evaluación financiera - fase diagnóstica}

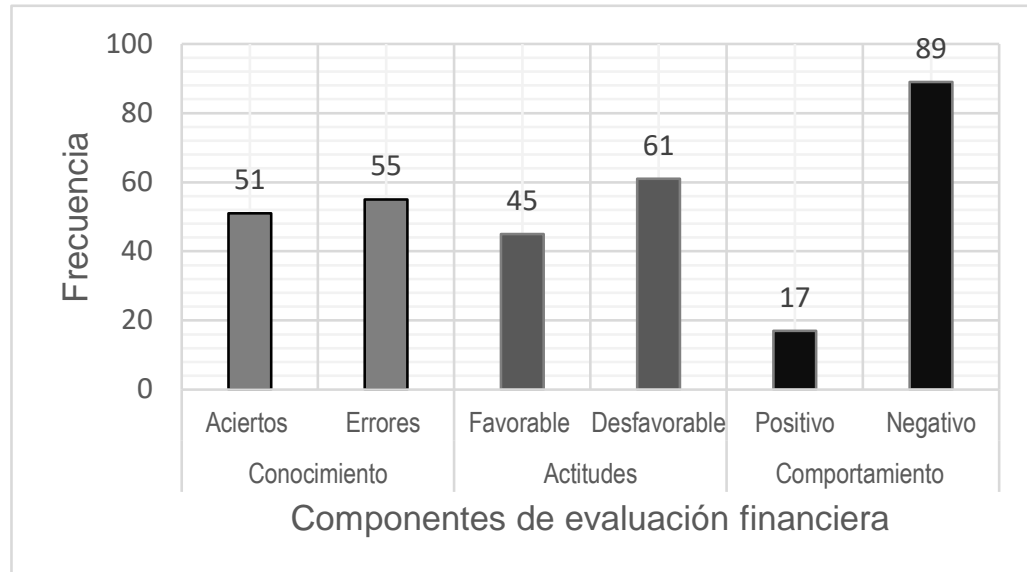

Fuente: test de diagnóstico

Elaborado por: las autoras

La aplicación del test de inteligencias múltiples de Gardner (2011) evalúa 7 diferentes tipos de inteligencias siendo estas: Inteligencia Verbal/ Lingüística, Inteligencia Lógico/ Matemática, Inteligencia Visual/ Espacial, Inteligencia Kinestésica/ Corporal, Inteligencia Musical/ Rítmica, Inteligencia Intrapersonal y la Inteligencia Interpersonal. En el ordenamiento se refleja la puntuación más alta obtenida que va de 1 a 5 en cada inteligencia siendo 5 la puntuación más alta en cada tipo de inteligencia.

Gráfico No. 5: A= Inteligencia Verbal/ Lingüística

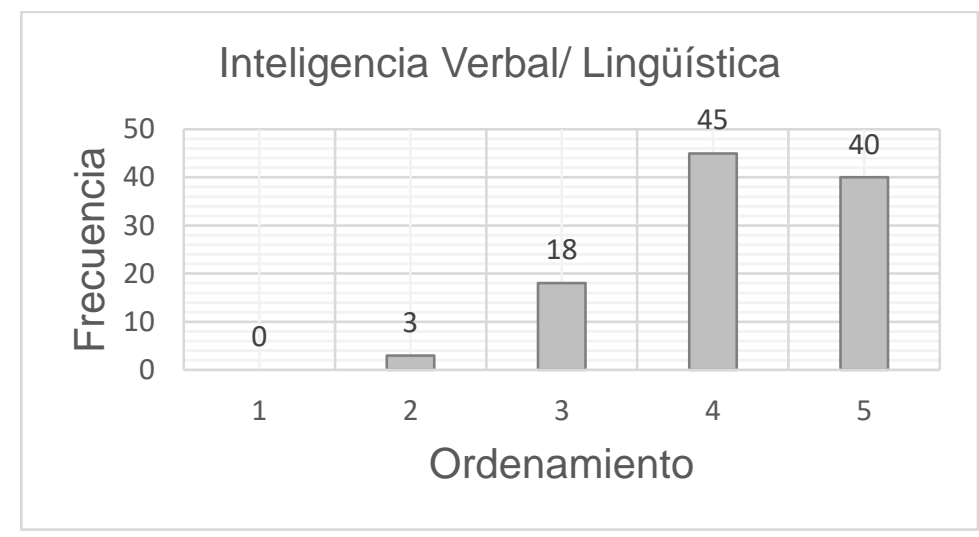

Fuente: test de diagnóstico

Elaborado por: las autoras 


\section{Gráfico No. 6: B= Inteligencia Lógico/ Matemática}

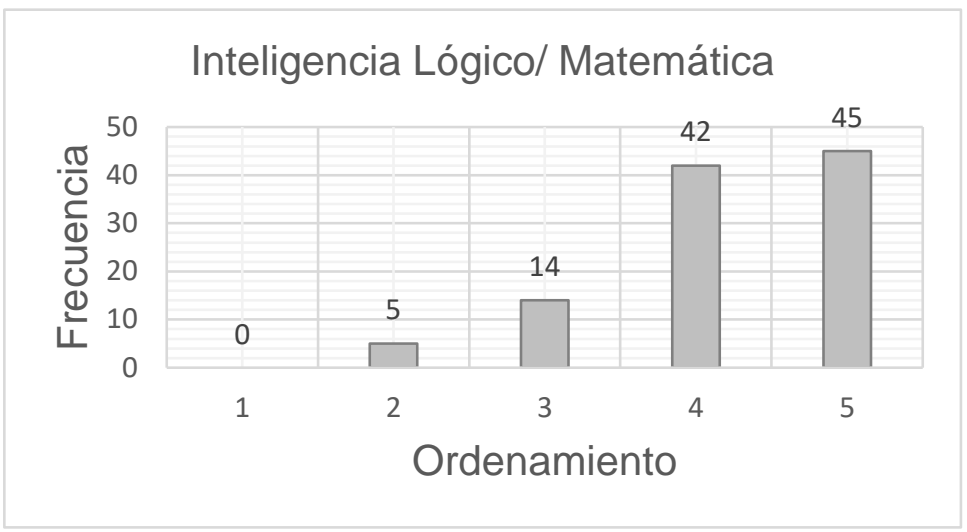

Fuente: test de diagnóstico Elaborado por: las autoras

\section{Gráfico No. 7: C= Inteligencia Visual/ Espacial}

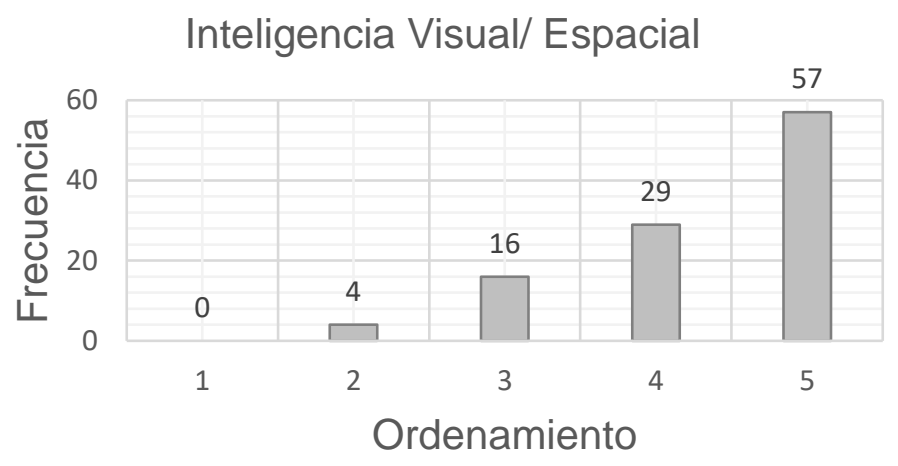

Fuente: test de diagnóstico

Elaborado por: las autoras

Gráfico No. 8: D= Inteligencia Kinestésica/ Corporal

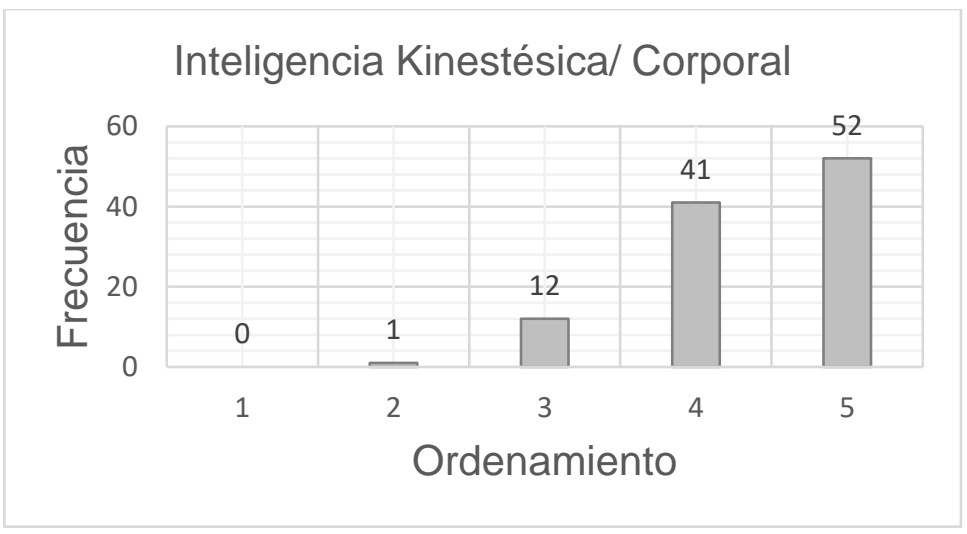

Fuente: test de diagnóstico

Elaborado por: las autoras 
Gráfico No. 9: E= Inteligencia Musical/ Rítmica

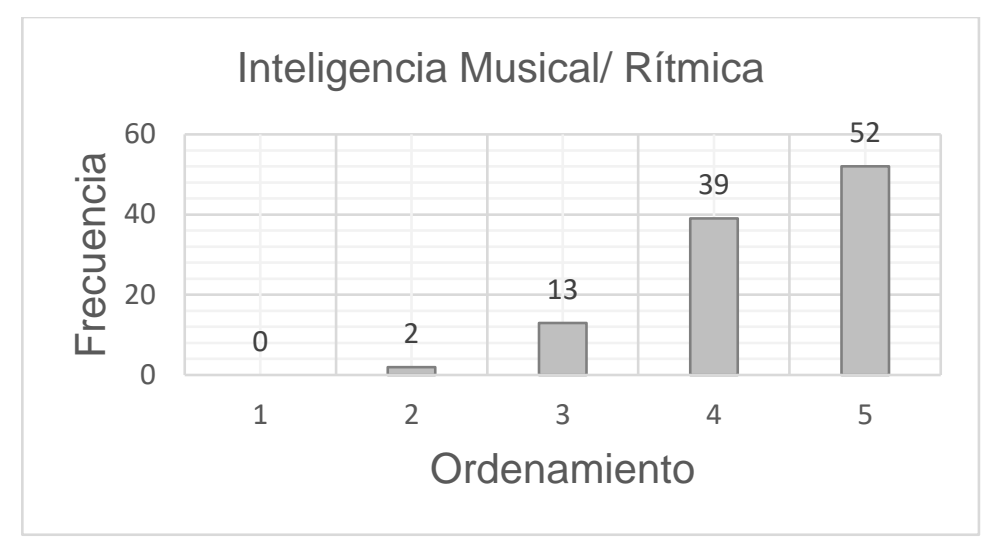

Fuente: test de diagnóstico

Elaborado por: las autoras

\section{Gráfico No. 10: F= Inteligencia Intrapersonal}

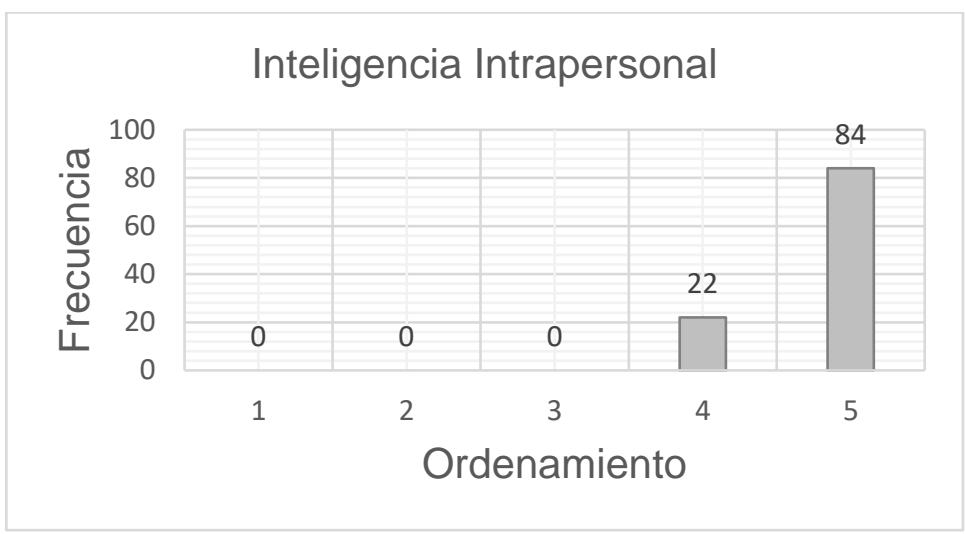

Fuente: trabajo de campo

Elaborado por: las autoras

\section{Gráfico No. 11: G= Inteligencia Interpersonal}

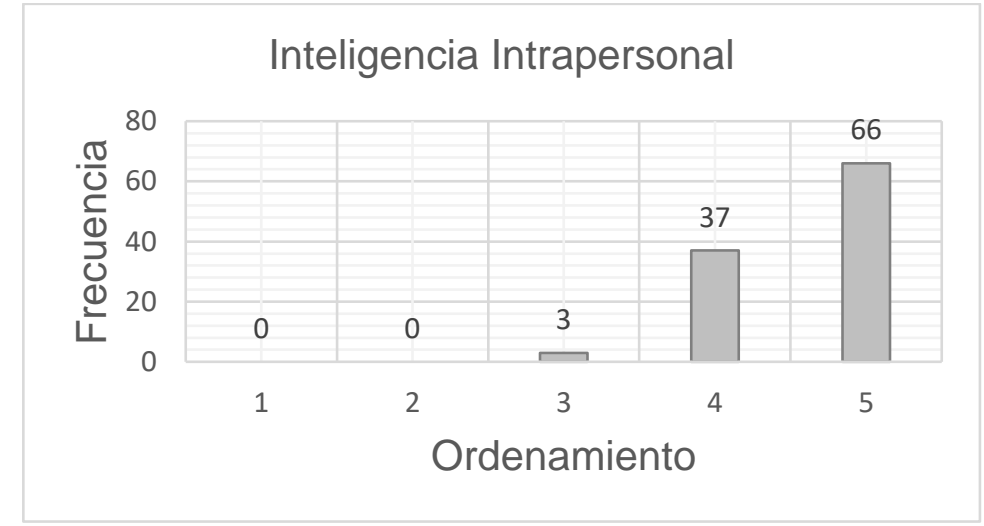

Fuente: test de diagnóstico

Elaborado por: las autoras 
La siguiente grafica muestra un consolidado del tipo de inteligencias que prevalece en el aula, identificándose 4 tipos predominantes: G (509), F (506), E (520) y D (474); y tres tipos de inteligencia con puntajes medianos: A (420), B (375), C (380).

\section{Gráfico No. 12: Inteligencias Múltiples observadas en el aula - fase diagnóstica}

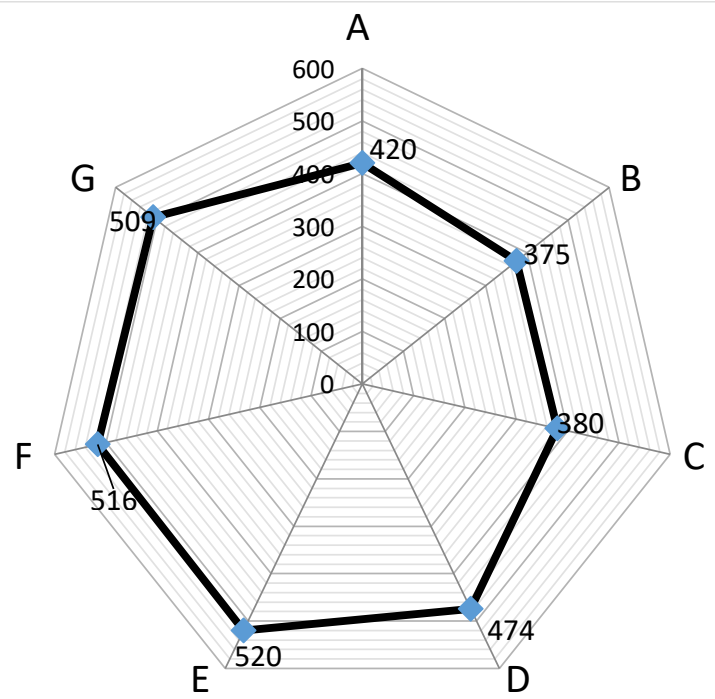

Inteligencias múltiples - fase diagnóstica

Fuente: test de diagnóstico

Elaborado por: las autoras

\section{Fase post - diagnóstica}

Una vez concluida la primera fase de diagnóstico, se procede ejecutar el programático (entrenamiento del OVA) y como última fase se aplica el test post- diagnostico utilizando los mismos instrumentos de evaluación, esta última etapa otorga resultados que validan la hipótesis planteada en principio del estudio.

\section{Gráfico No. 13: Componentes de evaluación financiera - fase post diagnóstica}

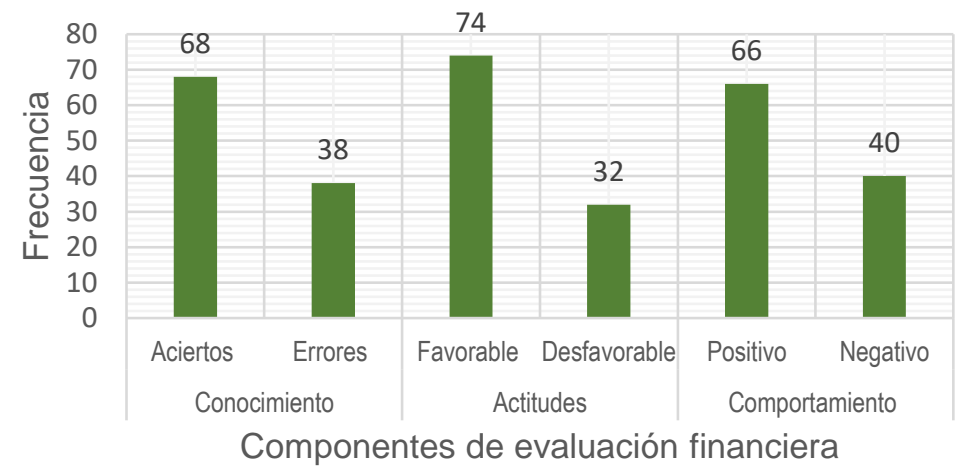

Fuente: test post diagnóstico

Elaborado por: las autoras 
La grafica muestra los resultados obtenidos de los componentes financieros después de haber aplicado el programático OVA, en el componente de conocimiento se distingue 68 aciertos contra 38 errores; en el componente aptitudes se observa 74 actitudes favorables contra 32 desfavorables y por último en el componente comportamiento se observa 66 comportamientos positivos contra 40 negativos.

\section{Gráfico No. 14: Inteligencias Múltiples observadas en el aula - fase post diagnóstica}

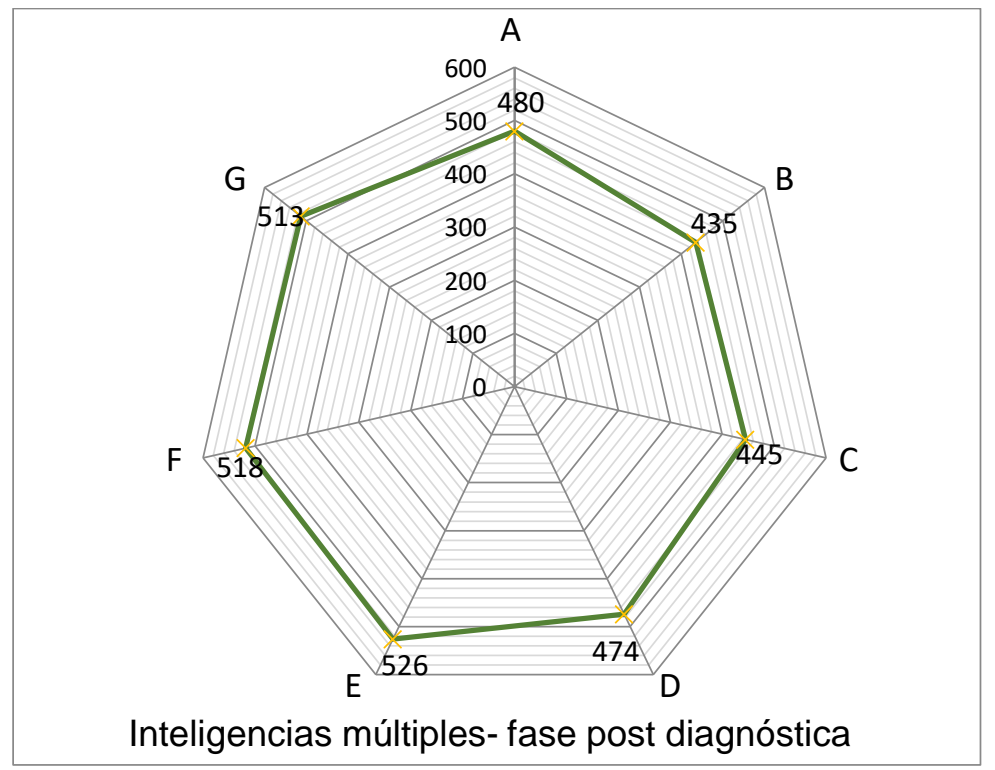

Fuente: test post diagnóstico

Elaborado por: las autoras

La grafica de las inteligencias múltiples de la fase post diagnostica presenta valores medianos siendo estos: G (513), F (518), E (526), D (474); A (480), B (435), C (445). Al comparar con la gráfica de diagnóstico la imagen actual, se presenta de manera casi uniforme con respecto a las diferentes inteligencias.

\section{Discusión.}

Los resultados de la prueba de fase de diagnóstico, en el componente de conocimientos técnicos financieros se evidencia un número considerable de error que supera los aciertos; las preguntas de este componente se relacionan con una de las inteligencias múltiples, la Inteligencia Lógico/ Matemática, que se caracteriza por tener la capacidad de identificar, calcular, formular teorías utilizando el razonamiento inductivo y deductivo. El resultado no es alentador pese a ser una base en la enseñanza de la carrera de administración financiera; en el componente actitudes financieras también se observa resultados desfavorables, las preguntas se relacionan con la Inteligencia Lógico/ Matemática e Inteligencia Lingüística, esta última se caracteriza por tener la capacidad de comprender el orden y significado de palabras; en referencia al componente de comportamiento financiero se destacan procedimientos negativos por gran parte de los estudiantes analizados, este componente se relaciona con las inteligencias: espacial caracterizada por tener la capacidad de percibir detalles visuales con precisión; inteligencia intrapersonal, evalúa ventajas y desventajas 
además de exhibir una diciplina personal y la inteligencia interpersonal tiene la capacidad de trabajar con gente e identificar y superar problemas.

En concordancia a los resultados iniciales, los conocimientos financieros no han sido suficientes para la mayoría de estudiantes, por lo tanto, las decisiones entorno al dinero no son acertadas, se diagnostica hechos de despreocupación, ausencia en establecimiento de metas financieras, escases en la búsqueda de información financiera; las malas experiencias en el manejo del dinero cohíben al estudiante a buscar nuevos retos a fin de llevar una económica personal efectiva.

En la fase post - diagnostico el estudiante desarrolla más ampliamente sus capacidades incentivado por la utilización de recursos tecnológicos como los OVAs, los resultados son considerables, hay una gran diferencia del $40 \%$ con el diagnóstico inicial. Una de las ventajas para el docente es la personalización de la herramienta, se puede adaptar de acuerdo a la capacidad de los estudiantes y al ritmo de aprendizaje, la constante utilización de las herramientas tecnológicas creara disciplina, conductas y comportamientos positivos en el aprendizaje. Conocer las habilidades del grupo con cualquier escala de medición no solo provee al docente información del ámbito académico, también predice las acciones de su entorno debido a las capacidades que posee. Con la aplicación objeto virtual de aprendizaje (CASHFLOW) que ofrece un escenario semejante a la realidad se comprueba la hipótesis planteada, incide efectivamente y despierta las capacidades del educando.

Ahora bien, la siguiente discusión considera las metodologías que el docente hace uso cuando ejerce la docencia en el aula, las entrevistas respaldan los puntos sobre la utilización inadecuada de herramientas tecnológicas, los resultados obtenidos del primer test sugieren un trabajo más arduo y completo; la motivación es otro factor prescindible se ha demostrado que esta acción lleva al estudiante a buscar más y mejores recursos para su subsistencia; es aquí donde el docente debe replantearse los métodos de enseñanza y evaluación que está aplicando, porque uno de los principios del docente es brindar una formación de calidad y calidez.

\section{Conclusiones.}

- La utilización de los recursos tecnológicos como los OVAs en el proceso de enseñanza - aprendizaje contribuye a mejorar las puntuaciones en el rendimiento académico y personal del estudiante.

- El aplicar metodologías de enseñanza-aprendizaje enmarcadas en la época actual facilitan y simplifican las actividades del docente en el aula.

\section{Referencias Bibliográficas}

Abarca, S. (1995). Psicología de la motivación. San José: Universidad.

BADA, S. O. (Diciembre de 2015). Teoría del aprendizaje del constructivismo: un paradigma para la enseñanza y Aprendizaje. IOSR Journal of Research \& Method in Education (IOSR-JRME), 66-70. doi:10.9790/7388-05616670 
Gardner, H. (2011). Inteligencias múltiples - La teoría en la práctica. Barcelona: Paidós.

Kaltakci, D., Eryllmaz, \& McDermot, L. (2015). Una revisión y comparación de instrumentos de diagnóstico para identificar estudiantes con conceptos erróneos en las ciencias. Eurasia Journal of Mathematics, Science \& Technology Education, 990991.

Kiyosaki, R. (3 de Diciembre de 2019). Rich Dad. Obtenido de The Rich Dad Company.: https://www.richdad.com/

Kiyosaki, R. T. (2012). Cuadrante de flujo de efectivo de Rich Dad: Guía de Rich Dad para la libertad financiera. (Vol. 2). Vietnam: Business Plus. doi:ISBN 9780446677479

Latorre, C. (19 de 10 de 2006). Objetos Virtuales de Aprendizaje. Obtenido de Unipanamericana:

http://aprendeenlinea.udea.edu.co/lms/men_udea/pluginfile.php/9322/mod_forum/at tachment/9946/OBJETOS_VIRTUALES_LECTURA.pdf.

MacDonald, K. (2019). Métodos y estrategias de enseñanza: la guía completa. EducationCorner.com.

Maldonado, J., Bermeo, J., \& Vélez, F. (2017). Diseño, Creación y Evaluación de Objetos de Aprendizaje - Metodología DICREVOA 2.0. Cuenca: CEDIA) Grafica Hernández.

Peel, E. A. (17 de mayo de 2017). Encyclopadia Britannica. Obtenido de Teorías de la enseñanza: psicología educativa: https://www.britannica.com/science/pedagogy

Sreenidhi, S. K., \& Tay, C. (2017). Evaluación de Inteligencia Múltiple Basado en la investigación de Howard Gardner. International Journal of Scientific and Research Publications Volume 7, Issue 4, 203-204. doi:ISSN 2250-3153

Wiley, D. (2000). Diseño de Objetos de Aprendizaje y teoría de secuenciación. Provo.

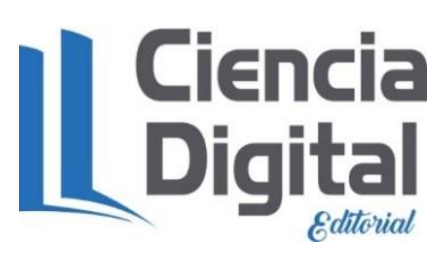




\section{PARA CITAR EL ARTÍCULO INDEXADO.}

Milla Estrada, A. de los Ángeles, \& Vázquez Andino, I. del R. (2020). Cashflow como objeto virtual de aprendizaje de la Administración Financiera. Caso: Instituto Luis A. Martínez de Ambato.

ConcienciaDigital, 3(4.1), 6-21. https://doi.org/10.33262/concienciadigital.v3i4.1.1466

El artículo que se publica es de exclusiva responsabilidad de los autores y no necesariamente reflejan el pensamiento de la Revista Conciencia Digital.

El artículo queda en propiedad de la revista y, por tanto, su publicación parcial y/o total en otro medio tiene que ser autorizado por el director de la Revista Conciencia Digital.

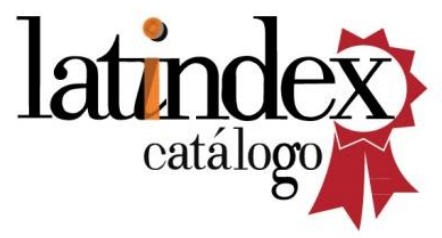

